\title{
Review
}

Respiration 2012;84:80-86

Received: August 30, 2011

DOI: $\underline{10.1159 / 000336238}$

Accepted after revision: December 24, 201

Published online: March 1, 2012

\section{Middle Lobe Syndrome: A Review of Clinicopathological Features, Diagnosis and Treatment}

\author{
Tomas Gudbjartsson ${ }^{\mathrm{a}, \mathrm{c}}$ Gunnar Gudmundsson ${ }^{\mathrm{b}, \mathrm{c}}$ \\ Departments of ${ }^{a}$ Cardiothoracic Surgery and ${ }^{b}$ Respiratory Medicine and Sleep, Landspitali - The National \\ University Hospital of Iceland, and ' Faculty of Medicine, University of Iceland, Reykjavik, Iceland
}

\section{Key Words}

Middle lobe syndrome - Review · Histopathology •

Pathophysiology $\cdot$ Bronchiectasis $\cdot$ Surgery $\cdot$ Radiology

\begin{abstract}
Middle lobe syndrome (MLS) is a rare but important clinical entity that has been poorly defined in the literature. It is characterized by recurrent or chronic collapse of the middle lobe of the right lung but can also involve the lingula of the left lung. Pathophysiologically, there are two forms of MLS, namely obstructive and nonobstructive. Obstructive MLS is usually caused by endobronchial lesions or extrinsic compression of the middle lobe bronchus such as from hilar lymphadenopathy or tumors of neoplastic origin, resulting in postobstructive atelectasis and pneumonitis. In the nonobstructive type, no obstruction of the middle lobe bronchus is evident during bronchoscopy or with computerized tomography of the chest. The etiology of the nonobstructive form is not completely understood. Inefficient collateral ventilation, infection and inflammation in the middle lobe or lingula are thought to play a role, and bronchiectasis is the most common histological finding. Patients with proven endobronchial lesions or malignancy are usually offered surgical resection directly. This contrasts with nonobstructive MLS, where most patients respond to medical treatment consisting of bronchodilators, mucolytics and broad-spec-
\end{abstract}

trum antibiotics. However, some patients do not respond to conservative treatment and may suffer irreversible damage of the middle lobe or lingula, in addition to having recurrent symptoms of infection or inflammation. These selected patients can be offered surgical resection of the middle lobe or lingula, which is associated with a low mortality rate and favorable outcome.

Copyright $\odot 2012$ S. Karger AG, Basel

\section{Introduction}

In 1948, Graham et al. [1] reported 12 patients with atelectasis and nontuberculous pneumonitis of the middle lobe of the right lung, which Effler and Ervin [2] subsequently termed the 'middle lobe syndrome' (MLS). There is limited literature on MLS, probably because of its multiple etiologies, varied presentation and lack of consistent clinical definition [3, 4]. The most common definition of MLS is recurrent or chronic collapse of the middle lobe of the right lung. Although originally described as occurring only in the middle lobe, the process can involve the lingula as well, which is sometimes called the lingula syndrome [5]. Pulmonary symptoms are common in MLS, but some patients are asymptomatic and their disease is only detected by chest radiography [6]. The epidemiology of MLS is not well described. It occurs

\section{KARGER}

Fax +4161306 1234

E-Mail karger@karger.ch

www.karger.com
(C) $2012 \mathrm{~S}$. Karger AG, Basel

0025-7931/12/0841-0080\$38.00/0

Accessible online at:

www.karger.com/res
Prof. Tomas Gudbjartsson, MD, $\mathrm{PhD}$

Department of Cardiothoracic Surgery

Landspitali - The National University Hospital of Iceland

13-C Hringbraut, IS-107 Reykjavik (Iceland)

Tel. +354 543 7755, E-Mail tomasgud@landspitali.is 
in children and adults of both sexes, in both primary and tertiary care settings $[7,8]$. Furthermore, no detailed information is available on the prevalence of the disease; however, in a study from Iceland involving the whole nation, the incidence of MLS requiring surgical resection - standardized to the WHO Europe (Scandinavia) population - was 1.43 per million males per year and 2.94 per million females per year [7].

\section{Etiology and Classification}

MLS can be divided pathophysiologically into obstructive and nonobstructive types. Obstructive MLS can be caused by endobronchial lesions or extrinsic compression of the right middle lobe bronchus. Tumors, both benign and malignant, have been estimated to account for about one quarter of cases of MLS [3,9], but it is important to note that many MLS studies have excluded patients with identifiable neoplasms $[1,3]$. Among the benign tumors that can cause obstruction are hamartomas, but malignant causes include primary lung cancer and metastases $[3,9]$. The most common cause of extrinsic compression of the right middle lobe bronchus is enlargement of the peribronchial lymph nodes [4]. This can be due to granulomatous infections such as histoplasmosis that cause adenopathy, but other fungal infections and typical and atypical mycobacterial infections are also well-described etiologies of obstructive MLS [3, 10-14]. Furthermore, adenopathy due to sarcoidosis or lymph node metastases has also been described in obstructive MLS [3]. Other less common causes of obstruction of the right middle lobe bronchus include aspirated foreign objects (particularly in children), broncholiths that erode endobronchially from adjacent calcified lymph nodes, inspissated mucus associated with cystic fibrosis or allergic bronchopulmonary aspergillosis, and endoluminal granulomas associated with sarcoidosis $[15,16]$. Some causes have been described as single case reports only [17-19].

In the nonobstructive type of MLS, the right middle lobe bronchus is patent with no demonstrable obstruction upon bronchoscopy $[7,8,20]$. This form of MLS is not restricted to the middle lobe and can be found in other lobes, most often the lingula of the left upper lobe [5]. The nonobstructive form has also been called peripheral MLS and occurs in the majority of patients with MLS $[3,6]$. It commonly occurs in adults and children with recurrent pneumonia and is often related to asthma, bronchitis and cystic fibrosis [7, 21, 22].

Middle Lobe Syndrome: Review

\section{Pathophysiology}

In the obstructive type of MLS, endobronchial lesions, foreign objects or extrinsic compression narrow the diameter of the lobar bronchus $[3,11,19]$. This obstructs the middle lobe bronchus, causing collapse of the middle lobe. The pathophysiology of nonobstructed MLS is more complex and less well defined. Certain anatomical characteristics make the middle lobe and lingula susceptible to transient obstruction as a result of inflammation or edema. This is probably related to the embryological development of the lungs [23]. At the early embryonic phase, the smaller left endodermal lung bud is directed more laterally than the caudally located right bud. Thus, the asymmetry of the main bronchi, as present in adults, is established early in lung development. At the next stage of development, the right main lung bud forms three further lung buds and the left one only two, corresponding to the later pulmonary lobes. The narrow diameter and long length of the middle lobar bronchus, combined with an acute take-off angle, create poor conditions for drainage $[3,5,8,23]$. Furthermore, the deep fissures of both the middle lobe and lingula, with only scanty parenchymal bridges, provide barriers to collateral ventilation [5]. This can contribute to poor clearance of mucus with peripheral plugging of the small airways $[19,23]$. The poor collateral ventilation of the middle lobe, especially in patients with complete fissures, and relative anatomical isolation reduce the chance of reinflation once atelectasis has occurred $[2,24]$. In some cases, no underlying lung disease can be found [23], as is often the case in the lingula syndrome.

\section{Epidemiology and Clinical Features}

MLS is more common in females; the ratio of females to males ranges from 1.5 to 3 in most studies $[3,4,6,8]$. The most common symptoms are chronic or recurrent cough, observed in $30-50 \%$ of patients $[3,7]$, dyspnea, chest pain, audible wheezing and fever and chills related to obstructive pneumonia $[3,7,20,25]$. These symptoms are often intermittent and recurrent. There is often a history of multiple treatments for recurrent pneumonia or asthma where antibiotics, mucolytics and bronchodilators have been used $[6,22,24]$. A family history of atopy is also common, and up to half of the patients report a history of atopy, asthma or chronic obstructive pulmonary disease $[3,8]$. Hemoptysis, weight loss, fatigue and low-grade fever are symptoms that may indicate compli-

Respiration 2012;84:80-86 
Fig. 1. Radiograph showing typical signs of MLS. a The lateral view shows the volume loss resulting from collapse of the right middle lobe (arrow). It can be seen as a triangle of increased density between the minor fissure and the lower half of the major fissure. Its apex is at the hilum, and the base is located peripherally towards the pleura. b The posteroanterior view shows obscuring of the right cardiac border (silhouette sign; arrow).
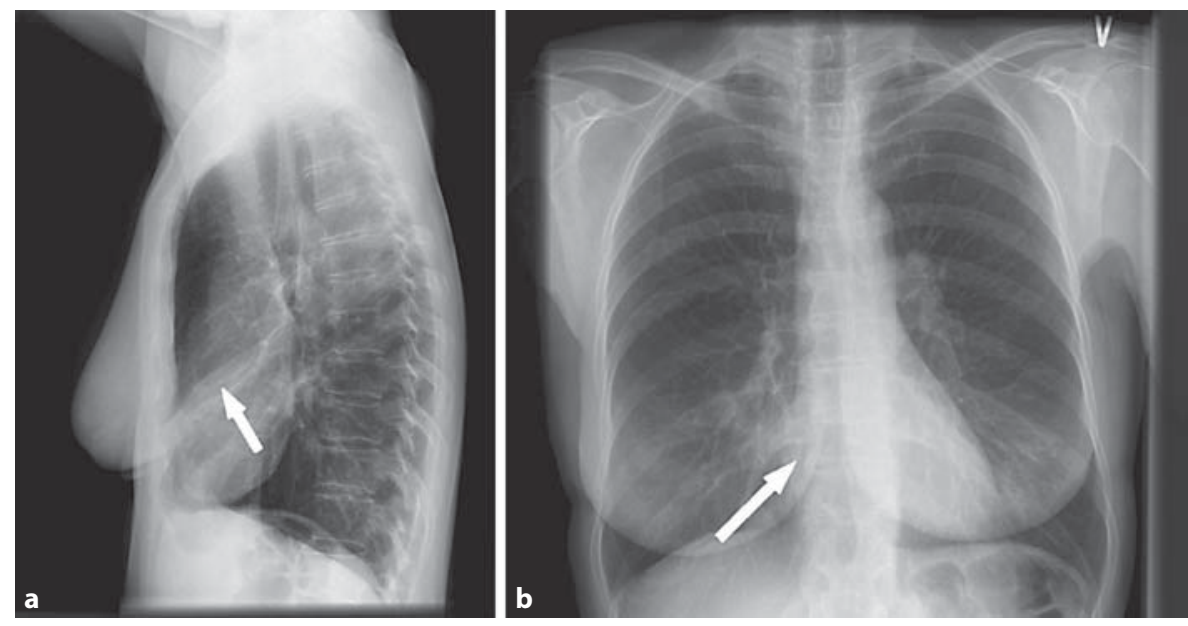

cations related to suppurative infections $[2,4,11]$. In rare cases, there are no symptoms and MLS is discovered by routine chest radiography [5]. Physical examination may be completely normal, but loss of breath sounds and vocal fremitus in the right middle lobe may also be apparent [2, $6,20]$. Patients with partial obstruction may have a palpable rhonchus, a localized wheeze on forced expiration and localized prolonged inspiratory time [19]. When performing auscultation of the chest, it is easy to miss the middle lobe if attention is not paid to its location $[19,22]$.

\section{Diagnostic Imaging}

Chest radiographs may be normal in patients with intermittent or recurrent collapse [6]. Most often, however, patients have abnormalities on the chest radiograph that are most apparent in the lateral view [5]. The volume loss resulting from collapse of the right middle lobe is seen as a triangle of increased density between the minor fissure and the lower half of the major fissure [5, 25] (fig. 1a). Its apex is at the hilum, and the base is peripherally located towards the pleura [19]. With long-standing collapse, this radiographic density may have a width of only $2-3 \mathrm{~mm}$ $[5,25]$. The posteroanterior radiograph may also show obscuring of the right cardiac border (silhouette sign) [5, 25] (fig. 1b) because the medial segment of the middle lobe is contiguous with the right atrium. Collapse of the middle lobe is often difficult to detect on the posteroanterior projection because the lobe is relatively thin and lies obliquely in the superoinferior plane $[5,25,26]$. The presence of air bronchograms suggests at least partial patency of the right middle lobe bronchus [5]. Calcified pa-

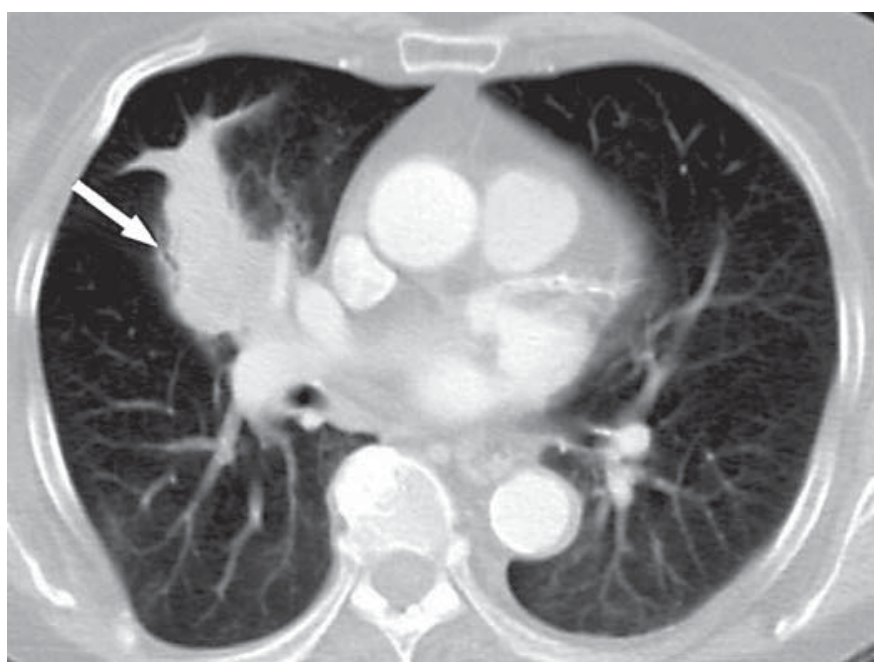

Fig. 2. A CT scan showing MLS with consolidation and atelectasis of the right middle lobe (arrow).

renchymal nodules and calcified hilar lymph nodes indicate a previous granulomatous infection $[6,26]$.

Plain chest radiographs do not usually reveal all the details that are necessary for the diagnosis or treatment of MLS $[5,26]$. Therefore, high-resolution, thin-section CT scans may be helpful, e.g. in identifying subtle endobronchial abnormalities, distal bronchiectasis and other parenchymal abnormalities [20,27]. Chest CT scans are also informative in evaluating bronchial patency, lymph node enlargement and calcifications or other causes of extrinsic compression of the right middle lobe airway [26]. They may also reveal a trapezoidal or broad triangular opacity within the base directed toward the hilum [26] 


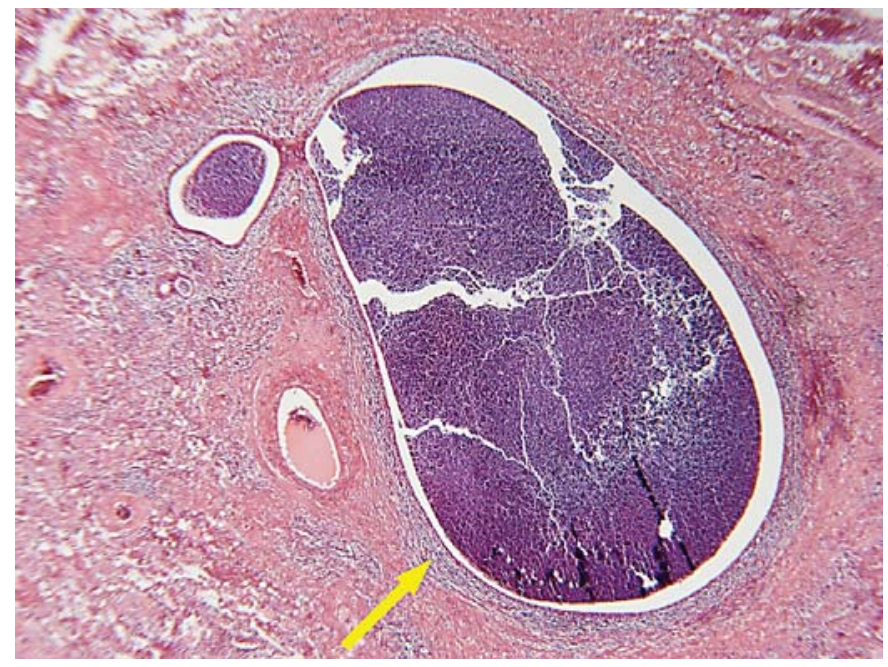

Fig. 3. A low-magnification photomicrograph showing bronchiectasis with dilated bronchi and peribronchial fibrosis (arrow). Hematoxylin and eosin staining.

(fig. 2). Typically, there is contiguity with part or all of the right cardiac border.

Flexible bronchoscopy (FB) is important in the evaluation of patients with MLS, especially regarding the patency of the right middle lobe bronchus [7, 11, 19]. In addition to excluding malignancy or other surgically correctable lesions, FB allows the collection of specimens for diagnosis of infectious causes [28]. Studies have shown that bronchoscopy findings are abnormal in up to $40 \%$ of cases $[7,9,11]$. Stenosis of the middle lobe bronchus and ostial tumors are the most commonly encountered abnormalities [29]. Endobronchial ultrasound can aid in the diagnosis of obstructive MLS by detecting lymph node enlargement and calcifications [30]. FB can also be helpful in children, where an unsuspected retained foreign body or benign tumor such as a hamartoma may predispose to recurrent infection [14, 21]. Other causes can also be found, such as bronchial hyperresponsiveness [31].

\section{Histopathology}

The major histological findings in MLS are bronchiectasis, which is found in up to $50 \%$ of patients, and foreign body reaction [7]. Less common findings are thrombi, arteriovenous malformations, chronic bronchial inflammation, pneumonia (acute and chronic), granulomatous inflammation and abscesses [7, 31, 32]. Exam- ples of pathology are shown in figure 3. Other histological findings include bronchiolitis, organizing pneumonia and peribronchial inflammation [20,32]. In one study in which pathological findings were described in 60 cases of MLS, 36 patients $(60 \%)$ were diagnosed as having chronic suppurations, 20 (33\%) as having neoplasia and 4 (7\%) as having tuberculosis [33]. However, as pointed out earlier, many MLS studies exclude patients with identifiable neoplasms $[1,3,19]$, and these patients usually do not have a prolonged clinical course with repeated episodes of antibiotics or other medications as they are operated on early [19].

\section{Treatment and Prognosis of MLS}

\section{Medical Therapy and Bronchoscopy}

Most cases of MLS receive treatment that is directed at the underlying cause, and this is true for both the obstructive and nonobstructive form. Patients with nonobstructive MLS usually respond to medical therapy with bronchodilators, mucolytic agents and antibiotics [20, 33]. Avoidance of bronchial irritants such as tobacco is also helpful [20]. MLS associated with asthma is treated with inhaled corticosteroids and bronchodilators [34]. Antibiotic treatment is usually recommended to attempt to eradicate an infection that can be associated with bronchiectasis [8]. However, the role of antibiotic therapy in the treatment of MLS is not well studied [3]. Antibiotic treatment is often continued for several weeks or months, as recurrences are common [19]. If available, antibiotics are chosen based on microbial culture or sensitivity results from bronchoalveolar lavage fluid or sputum [11-13, 27]. Otherwise, broad-spectrum antibiotics are used, covering streptococci (including Streptococcus pneumoniae), Haemophilus influenzae, Moraxella catarrhalis and even Pseudomonas aeruginosa in difficult cases [3]. Low-dose macrolide therapy can be used, particularly if bronchiectasis is present [35]. Consideration must also be given to unusual infections (atypical mycobacteria, fungi) that may require specific antimicrobial agents $[11,12$, 14]. The use of nebulized antibiotics has not been studied in MLS.

Nonsurgical techniques using FB to treat specific cases of MLS have been described. Techniques designed to increase mean air pressure in the right middle lobe bronchus, such as bronchoscopically directed air insufflation or noninvasive positive pressure ventilation, remain intriguing, but largely unexplored, therapeutic interventions. Selective intrabronchial air insufflation us- 
ing specially outfitted FB has been used to treat lobar collapse [36]. For the obstructive form, broncholiths and foreign objects have been removed with $\mathrm{FB}$ or rigid bronchoscopy $[29,37]$. Other nonsurgical bronchoscopic options are balloon dilatation, stent placement, argon plasma coagulation, electrocautery, cryosurgery and laser therapy to relieve focal nonmalignant obstructions $[29,38-40]$.

Flutter valve and high-frequency oscillation (known as the vest) have not been studied in MLS, but they may be alternative modalities of delivering chest physical therapy. Chest physical therapy and postural drainage are thought to be of importance in the management of MLS [41]. This is often performed by a registered respiratory or physical therapist.

There are no data from randomized, controlled studies to determine the proportion of patients that require surgery because conservative treatment has failed. In addition, the role of FB and antibiotics in the management of MLS is constantly evolving. This is especially true for pediatric patients. In a study on 17 children diagnosed with MLS in early childhood (with an average age of 3.3 years), about one third continued to have respiratory symptoms in later childhood, most commonly mild obstructive airway disease [42]. None of these children had been operated on during a mean follow-up time of 10.1 years. Furthermore, chronic lower respiratory tract infection only occurred in one patient, supporting conservative management in most patients [3, 42]. In another study, Priftis et al. [41] evaluated 55 children with MLS (mean age 5.5 years), 2 of whom had bronchial obstruction. Two years later, 49 of the 53 patients (92\%) with nonobstructive MLS were cured or their symptoms had improved. This was achieved with early FB and therapeutic bronchoalveolar lavage. This strategy was believed to prevent later development of bronchiectasis if instituted within 3-7 months from the start of deterioration of symptoms [41]. In a study by Livingston et al. [15], a cure rate of $33 \%$ with conservative treatment was achieved. Bronchoscopy played an important role, and only one third of patients required surgical resection [15]. Furthermore, in a study on 98 adult patients with MLS, Saha et al. [43] reported that only $32 \%$ required surgical resection for persistent disease.

\section{Surgical Intervention}

Indications and Timing of Surgery

Surgical removal of the middle lobe is reserved for resistant and complex cases of MLS, usually for patients with isolated MLS who do not respond to medical ther- apy and who have proven obstruction of the middle lobe bronchus [8]. Currently, there is no consensus regarding the indication for surgical removal of the middle lobe in MLS. There have been few studies, most of them not randomized and with a small number of patients $[5,32]$. Many of these studies were also limited to children, where MLS cases were analyzed together with bronchiectasis of the lingula [5]. Larger studies on surgical treatment of adult bronchiectasis have been published; however, most patients in these studies had diffuse bronchiectasis, with disease that was not confined to the middle lobe $[44,45]$.

Recurrent hemoptysis in a patient with MLS in whom interventional radiology techniques have not been successful is an indication for surgery [46]. If biopsy or bronchial washings suggest malignancy in the middle lobe, surgery is indicated for the malignancy. Under such circumstances, the middle lobe is removed together with surrounding hilar and mediastinal lymph nodes [47]. Surgery may also be indicated in patients with MLS who have scarring, fibrosis or abscess formation $[8,20]$.

In nonobstructive MLS, the timing of surgical treatment is difficult to decide. Lobectomy is only considered if symptoms persist with radiographic evidence of chronic atelectasis after prolonged medical therapy, usually for at least 6 months. The surgical procedure is usually more successful if the disease is limited to the middle lobe only $[45,46]$. In children, surgical resection is only advocated when symptoms are debilitating, such as persistent cough, failure to thrive or recurrent fever [5, 39]. Other studies in children have advocated early pulmonary resection in the presence of bronchiectasis, bronchial stenosis or failure of the lung to re-expand $[3,32]$.

\section{Surgical Outcome}

Results after surgery for MLS are poorly documented, especially in adults. Recently, we studied 18 patients with MLS, 17 of them adults, who were treated with open lobectomy [8]. Peri- and postoperative complications were usually minor, and no major complications occurred. Bleeding was the most common complication (11\%) and was usually related to difficult dissection of hilar structures secondary to extensive inflammation of the middle lobe. Other complications, such as extensive air leakage (11\%) and chronic atelectasis of the inferior lobe (6\%), were similar to complications seen after lobectomy for non-small cell lung cancer $[48,49]$. Only 1 patient was reoperated, for both bleeding and air leakage. All 18 patients survived the operation, and 5- and 10 -year postoperative crude survival was 94 and $83 \%$, respectively, with 
no patients dying from pulmonary-related disorders [8]. Out of 18 patients, 13 (72\%) were cured of their symptoms and 5 other patients were improved. Similar results have been reported in other studies, including the study by McElvein and Mayo [25], in which 21 of 22 patients (95\%) were free of symptoms after surgery. In another study, by Bradham et al. [10], all of the 41 surgically treated patients were able to perform normal activities following surgery, and 32 (78\%) were free of symptoms. Furthermore, in their report, no deaths or significant complications followed surgery for MLS [10]. In a study on 13 children with MLS, Ayed [5] reported 8 asymptomatic patients after surgery, with 2 others having evidence of mild bronchiectasis in the opposite lung. Recently, thoracoscopic lobectomy was reported in 3 children as a therapeutic alternative in persistent MLS, with promising results [50].

\section{Conclusion}

Many patients with MLS, especially those with the nonobstructive form, respond to medical treatment consisting of bronchodilators, mucolytics, FB and broad-spectrum antibiotics. However, patients who do not respond to conservative treatment can be offered surgical resection with removal of the middle lobe or lingula. Although based on a limited number of studies, operative mortality and morbidity appear to be low following these procedures, and long-term outcome appears to be favorable.

\section{Acknowledgements}

We thank Olafur Baldursson, MD, PhD, for comments, Helgi J. Isaksson, $\mathrm{MD}$, for providing histopathology photographs, and Hjalti Mar Thorisson, MD, for providing radiographs.

\section{Financial Disclosure and Conflicts of Interest}

\section{References}

1 Graham EA, Burford TH, Mayer JH: Middle lobe syndrome. Postgrad Med 1948;4:29-34.

$\checkmark 2$ Effler DB, Ervin JR: The middle lobe syndrome; a review of the anatomic and clinical features. Am Rev Tuberc 1955;71:775-784.

3 Bertelsen S, Struve-Christensen E, Aasted A, Sparup J: Isolated middle lobe atelectasis: aetiology, pathogenesis, and treatment of the so-called middle lobe syndrome. Thorax 1980;35:449-452.

4 Culiner MM: The right middle lobe syndrome, a non-obstructive complex. Dis Chest 1966;50:57-66.

5 Ayed AK: Resection of the right middle lobe and lingula in children for middle lobe/lingula syndrome. Chest 2004;125:38-42.

6 Fraser R, Pare J, Pare P, Frazer R, Generoux G: Roentgenologic signs in the diagnosis of chest disease; in Fraser RG, et al (eds): Diagnosis of Diseases of the Chest. Philadelphia, Saunders, 1988

7 Dees SC, Spock A: Right middle lobe syndrome in children. JAMA 1966;197:8-14.

$\checkmark 8$ Einarsson JT, Einarsson JG, Isaksson $\mathrm{H}$, Gudbjartsson T, Gudmundsson G: Middle lobe syndrome: a nationwide study on clinicopathological features and surgical treatment. Clin Respir J 2009;3:77-81.

-9 Wagner RB, Johnston MR: Middle lobe syndrome. Ann Thorac Surg 1983;35:679-686.
0 Bradham RR, Sealy WC, Young WG Jr: Chronic middle lobe infection. Factors responsible for its development. Ann Thorac Surg 1966;2:612-616.

11 Kala J, Sahay S, Shah A: Bronchial anthracofibrosis and tuberculosis presenting as a middle lobe syndrome. Prim Care Respir J 2008; 17:51-55.

12 Kim HC, Kim HS, Lee SJ, et al: Endobronchial tuberculosis presenting as right middle lobe syndrome: clinical characteristics and bronchoscopic findings in 22 cases. Yonsei Med J 2008;49:615-619.

13 Kinzy JD, Powers WP, Baddour LM: Case report: Blastomyces dermatitidis as a cause of middle lobe syndrome. Am J Med Sci 1996; 312:191-193.

14 Lambert GW, Baddour LM: Right middle lobe syndrome caused by Mycobacterium fortuitum in a patient with human immunodeficiency virus infection. South Med J 1992; 85:767-769.

15 Livingston GL, Holinger LD, Luck SR: Right middle lobe syndrome in children. Int J Pediatr Otorhinolaryngol 1987;13:11-23.

16 Oshima M, Maeda H, Furonaka O, Doi M, Nishizaka T, Kuwabara M: Sarcoidosis with multiple organ involvement emerging as Lofgren's syndrome. Intern Med 2003;42: 534-537.
17 Chen HA, Lai SL, Kwang WK, Liu JC, Chen $\mathrm{CH}$, Huang DF: Middle lobe syndrome as the pulmonary manifestation of primary Sjogren's syndrome. Med J Aust 2006;184:294295.

18 Chien HP, Lin TP, Chen HL, Huang TW: Right middle lobe atelectasis associated with endobronchial silicotic lesions. Arch Pathol Lab Med 2000;124:1619-1622.

19 Illamperuma C, Reid J, Kanthan R: Chyloptysis with right middle lobe syndrome complicated postoperatively by chylothorax: an unusual cause of right middle lobe syndrome. Can Respir I 2009; 16:e1-e2.

20 Gudmundsson G, Gross TJ: Middle lobe syndrome. Am Fam Physician 1996;53:25472550.

21 Springer C, Avital A, Noviski N, et al: Role of infection in the middle lobe syndrome in asthma. Arch Dis Child 1992;67:592-594.

22 Thacher H, Kaplan A: Middle lobe syndrome in asthmatic children. J Maine Med Assoc 1972;63:46-48 passim.

-23 Berrocal T, Madrid C, Novo S, Gutierrez J, Arjonilla A, Gomez-Leon N: Congenital anomalies of the tracheobronchial tree, lung, and mediastinum: embryology, radiology, and pathology. Radiographics 2004;24:e17.

24 Inners CR, Terry PB, Traystman RJ, Menkes HA: Collateral ventilation and the middle lobe syndrome. Am Rev Respir Dis 1978;118: 305-310. 
25 McElvein RB, Mayo P: Middle lobe disease. South Med J 1967;60:1029-1032.

26 Rosenbloom SA, Ravin CE, Putman CE, et al: Peripheral middle lobe syndrome. Radiology 1983;149:17-21.

27 Javidan-Nejad C, Bhalla S: Bronchiectasis. Radiol Clin North Am 2009;47:289-306.

28 Rock MJ: The diagnostic utility of bronchoalveolar lavage in immunocompetent children with unexplained infiltrates on chest radiograph. Pediatrics 1995;95:373377.

29 Erelel M, Yakar F, Yakar A: Endobronchial tuberculosis with lobar obstruction successfully treated by argon plasma coagulation. South Med J 2009;102:1078-1081.

30 Becker H, Herth F: Endobronchial ultrasound of the airway and the mediastinum; in Bolliger C, Mathur P (eds): Interventional Bronchoscopy. Prog Respir Res. Basel, Karger, 2000, vol 30, pp 80-93.

- 31 Priftis KN, Anthracopoulos MB, Mermiri D, et al: Bronchial hyperresponsiveness, atopy, and bronchoalveolar lavage eosinophils in persistent middle lobe syndrome. Pediatr Pulmonol 2006;41:805-811.

32 Kwon KY, Myers JL, Swensen SJ, Colby TV: Middle lobe syndrome: a clinicopathological study of 21 patients. Hum Pathol 1995;26: 302-307.

-33 Eskenasy A, Eana-Iorgulescu L: Pathology of the middle lobe syndromes. A histopathological and pathogenetic analysis of sixty surgically-cured cases. Med Interne 1982;20: 73-80.
34 Sekerel BE, Nakipoglu F: Middle lobe syndrome in children with asthma: review of 56 cases. J Asthma 2004;41:411-417.

35 Kawamura M, Arai Y, Tani M: Improvement in right lung atelectasis (middle lobe syndrome) following administration of lowdose roxithromycin. Respiration 2001;68: 210-214.

36 Wohlauer MV, Moore EE, Haenel JB, Burlew CC, Barnett CC: Selective intrabronchial air insufflation for acute lobar collapse in the surgical intensive care unit. J Surg Radiol 2011;2:178-180.

37 Pasaoglu I, Dogan R, Demircin M, et al: Bronchoscopic removal of foreign bodies in children: retrospective analysis of 822 cases. Thorac Cardiovasc Surg 1991;39:95-98.

38 Sutedja G, Bolliger CT: Endobronchial electrocautery and argon plasma coagulation; in Bolliger CT, Mathur PN (eds): Interventional Bronchoscopy. Prog Respir Res. Basel, Karger, 2000, vol 30, pp 120-132.

39 Mathur PN, Wolf KM, Busk MF, Briete WM, Datzma M: Fiberoptic bronchoscopic cryotherapy in the management of tracheobronchial obstruction. Chest 1996;110:718-723.

40 Freitag L: Tracheobronchial stents; in Bolliger CT, Mathur PN (eds): Interventional Bronchoscopy. Prog Respir Res. Basel, Karger, 2000, vol 30, pp 171-186.

41 Priftis KN, Mermiri D, Papadopoulou A, Anthracopoulos MB, Vaos G, Nicolaidou P: The role of timely intervention in middle lobe syndrome in children. Chest 2005;128: 2504-2510.
42 De Boeck K, Willems T, Van Gysel D, Corbeel L, Eeckels R: Outcome after right middle lobe syndrome. Chest 1995;108:150-152.

43 Saha SP, Mayo P, Long GA, McElvein RB: Middle lobe syndrome: diagnosis and management. Ann Thorac Surg 1982;33:28-31.

44 Corless JA, Warburton CJ: Surgery vs. nonsurgical treatment for bronchiectasis. CochraneDatabaseSystRev2000;(4):CD002180.

45 Dogan R, Alp M, Kaya S, et al: Surgical treatment of bronchiectasis: a collective review of 487 cases. Thorac Cardiovasc Surg 1989;37: 183-186.

46 O’Donnell AE: Bronchiectasis. Chest 2008; 134:815-823.

-47 Scott WJ, Howington J, Feigenberg S, Movsas B, Pisters K; American College of Chest Physicians: Treatment of non-small cell lung cancer stage I and stage II: ACCP evidencebased clinical practice guidelines (2nd edition). Chest 2007;132(3 suppl):234S-242S.

- 48 Myrdal G, Gustafsson G, Lambe M, Horte LG, Stahle E: Outcome after lung cancer surgery. Factors predicting early mortality and major morbidity. Eur J Cardiothorac Surg 2001;20:694-699.

49 Skuladottir R, Oskarsdottir GN, Isaksson HJ, Jonsson S, Thorsteinsson H, Gudbjartsson T: Postoperative complications following lobectomy for lung cancer in Iceland during 1999-2008 (in Icelandic). Laeknabladid 2010;96:243-249.

50 Seitz G, Warman SW, Szavay PO, et al: Thoracoscopic lobectomy as a treatment option for persistent middle lobe syndrome in children. J Pediatr Int 2010;52:79-81. 